\title{
Eculizumab dosing regimen in atypical HUS: possibilities for individualized treatment
}

Elena Volokhina ${ }^{\mathrm{a}, \mathrm{b}}$, Kioa Wijnsma ${ }^{\mathrm{a}}$, Renate van der Molen ${ }^{\mathrm{b}}$, Nel Roeleveld $^{\mathrm{a}, \mathrm{c}}$, Thea van der Velden ${ }^{\mathrm{a}}$, Joop Goertz ${ }^{\mathrm{b}}$, Fred Sweep ${ }^{\mathrm{b}}$, Roger J Brüggemann ${ }^{\mathrm{d}}$, Jack Wetzels ${ }^{\mathrm{e}}$, Nicole van de Kar ${ }^{\mathrm{a}}$, Lambertus van den Heuvel ${ }^{\mathrm{a}, \mathrm{b}, \mathrm{f}}$

Departments of ${ }^{a}$ Pediatric Nephrology, Amalia Children's Hospital, ${ }^{b}$ Laboratory Medicine, ${ }^{c}$ Health Evidence, ${ }^{d}$ Pharmacy and ${ }^{e}$ Nephrology, Radboud university medical center, P. O. Box 9101, 6500 HB, Nijmegen, The Netherlands; ${ }^{f}$ Department of Pediatrics, University Hospitals Leuven, Herestraat 49, 3000 Leuven, Belgium

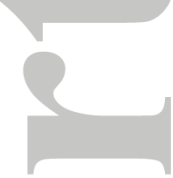

\section{Corresponding author:}

Prof. dr, L.P. van den Heuvel

Department of Pediatric Nephrology (830), Radboud university medical center

P.O. Box 9101, 6500 HB Nijmegen, The Netherlands

Tel: +31-24-3614567

Email: Bert.vandenHeuvel@radboudumc.nl

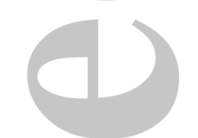

Key words: nephrology, monoclonal antibodies, dose, optimization, pharmacokineticspharmacodynamics

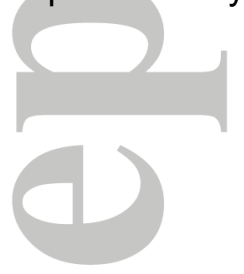

References: 48

Figures: $\quad 4$

Tables: $\quad 2$

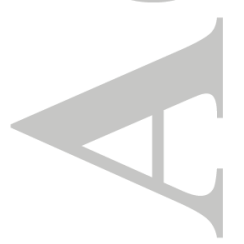

This article has been accepted for publication and undergone full peer review but has not been through the copyediting, typesetting, pagination and proofreading process which may lead to differences between this version and the Version of Record. Please cite this article as an 'Accepted Article', doi: 10.1002/cpt.686 


\section{Abstract}

Recent studies indicate that eculizumab is often given in excess to aHUS patients. Individualization of treatment is thus highly requested, however, data on pharmacokinetics and pharmacodynamics of eculizumab remain limited. We analyzed 11 patients during induction (weekly), maintenance (2-weekly) and tapering (every 3-8 weeks) phases of treatment.

The trough eculizumab levels increased with each additional dose during the induction phase (depending on body weight). During maintenance, high eculizumab concentrations of up to $772 \mu \mathrm{g} / \mathrm{mL}$ were observed. The levels decreased with each following dose during tapering (3- and 4-week intervals), however three patients maintained target eculizumab levels over long time periods (30-48 weeks). At intervals of 6-8 weeks target eculizumab levels were no longer attained. Serum samples with eculizumab concentrations $\geq 50 \mu \mathrm{g} / \mathrm{mL}$ showed adequate complement blockade.

Our data provide essential insight for optimization of eculizumab dosing schemes and lessening of therapy burden for the patients and cost of the treatment.

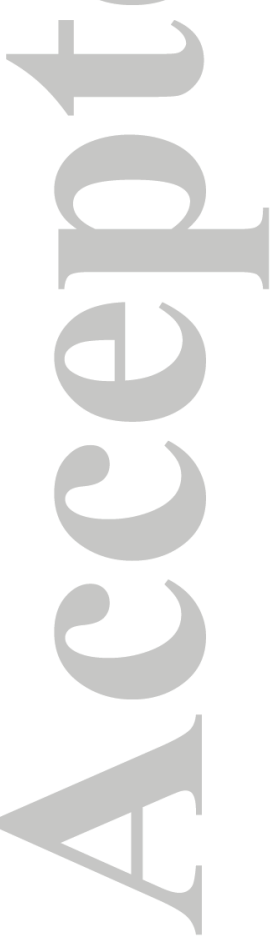




\section{Introduction}

Hemolytic uremic syndrome (HUS) is a severe form of thrombotic microangiopathy

(TMA) characterized by hemolytic anemia, thrombocytopenia and acute renal failure. The majority of cases are caused by an infection with Shiga toxin producing Escherichia coli (STEC). However, $5-10 \%$ of HUS occurs without a preceding STEC infection and is attributed to complement dysregulation [1-6]. Currently, alternative complement pathway aberrations are identified in $50-60 \%$ of atypical HUS (aHUS) patients. Genetic variants affecting complement factor $\mathrm{H}(\mathrm{CFH})$, complement factor I (CFI), membrane co-factor protein (CD46), C3, complement factor B and thrombomodulin as well as autoantibodies against CFH (anti-CFH) are associated with aHUS pathogenesis [7-19]. More recently, genetic rearrangements resulting in $\mathrm{CFH} / \mathrm{CFH}$-related hybrid proteins have also been described [2022].

Eculizumab is a humanized monoclonal complement inhibitor that binds to $\mathrm{C} 5$ and prevents its cleavage and activation into $\mathrm{C} 5 \mathrm{a}$ and $\mathrm{C} 5 \mathrm{~b}$, blocking formation of the terminal complement complex C5b-9. Eculizumab is currently approved by the Food and Drug Administration and European Medicines Agency (EMA) for the treatment aHUS and has proven to be highly efficient in the treatment of this disease [5, 23-27]. The current international guidelines recommend life-long treatment with eculizumab. However, the resulting life-long complement blockade may increase the risk of meningococcal infection, and eculizumab treatment is associated with extreme costs [28, 29]. There are no studies that would support the necessity of a life-long treatment in all aHUS patients. Before eculizumab has become available, plasma therapy was a main-stream approach for the treatment of aHUS. The guidelines for plasma therapy in aHUS advised individualized regimen and withdrawal in patients that have reached remission [30, 31]. Therefore, many nephrologists use a restrictive approach for eculizumab treatment in aHUS and discontinue therapy when patients reach remission [32-39]. A subset of patients that discontinued eculizumab in these reports experienced relapse, and the therapy had to be reinitiated. This underscores the need for close monitoring of these patients for signs of relapse. 
The recommended concentrations of eculizumab for efficient complement blockade are $50-100 \mu \mathrm{g} / \mathrm{ml}$, however in the past our and other studies demonstrated that eculizumab levels in patients, that receive the drug every two weeks exceed this reference by up to ten fold [32, 40-42]. Thus, optimization of dosing regimen in eculizumab therapy is urgently needed, however, pharmacokinetic and pharmacodynamic data to guide such personalized treatment schemes remain limited.

In this study, we followed patients who were treated with standard and extended infusion intervals, and analyzed eculizumab concentrations and complement blockade in these patients.
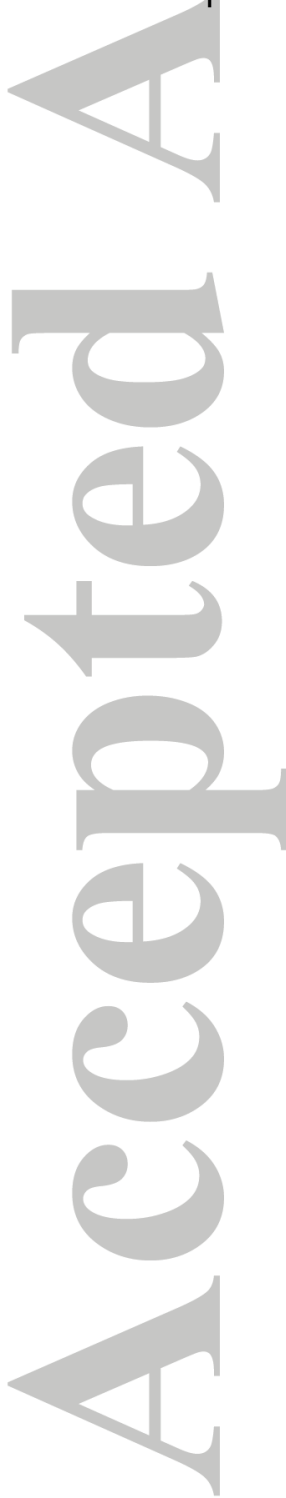


\section{Results}

\section{Patient cohort}

In total, five pediatric and six adult patients diagnosed with aHUS treated with eculizumab were included in this study (Table 1). In nine of these patients, pathogenic variations in the complement genes were found. One aHUS patient (P3) had anti-CFH and in one other patient (P9) no genetic abnormalities or anti-CFH were detected. In nine patients, the intervals between eculizumab infusions were extended for up to eight weeks after stable remission was achieved. In one of these patients (P1), eculizumab treatment was discontinued completely. Serum samples of this patient were continuously collected through week 26 after the last eculizumab dose (Table 1), no disease recurrence was observed during the study period. In two patients, eculizumab treatment was discontinued without extension of dosage intervals beyond two weeks due to an allergic reaction (P8) and severe pneumonia (P11).

\section{Serum eculizumab concentrations in aHUS cohort}

The eculizumab trough concentrations (lowest drug concentration in blood between two consecutive infusions) that were measured in 11 patients are depicted in Figure $1 \mathrm{~A}$. The detected values were $36-459 \mu \mathrm{g} / \mathrm{mL}(\mathrm{n}=27)$ and $40-772 \mu \mathrm{g} / \mathrm{mL}(\mathrm{n}=90)$ during the induction and maintenance phases, respectively. During tapering, concentrations of $61-367 \mu \mathrm{g} / \mathrm{mL}$ $(\mathrm{n}=38), 11-256 \mu \mathrm{g} / \mathrm{mL}(\mathrm{n}=38)$ and 13-161 $\mu \mathrm{g} / \mathrm{mL}(\mathrm{n}=6)$ were measured at 3- , 4- and 5- week infusion intervals. At intervals of 6-8 weeks $(n=10)$, the concentrations were $0-41 \mu \mathrm{g} / \mathrm{mL}$. For infusion intervals of $1-5$ weeks, attainment of the minimal target level of $50 \mu \mathrm{g} / \mathrm{mL}$ was found in $75-91 \%$ of patients (Figure 1B).

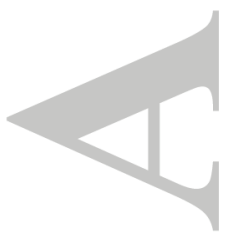




\section{Serum eculizumab concentrations in individual patients during infusions with}

standard and extended intervals

In the induction phase, the eculizumab levels gradually increased with each following 1-week interval by on average $73.1(95 \% \mathrm{Cl}: 56.2-90.0) \mu \mathrm{g} / \mathrm{mL}$, adjusted for body weight (Table 2). Two adult patients (P10 and P11) did not attain the target eculizumab level after the first infusion, but did so after the second dose (Figure 2A). Figure 3 shows that patients P10 and P11 had the lowest trough eculizumab concentrations one week after the first eculizumab infusion and the highest sC5b-9 complex levels measured before the first infusion. The serum C5 levels in patients in acute phase before start of therapy were 52-136

$\mu \mathrm{g} / \mathrm{mL}$, while values obtained from nine healthy controls were $80-107 \mu \mathrm{g} / \mathrm{mL}$.

Serum eculizumab concentrations did not change with each following dose at 2-week intervals (Figure 2B, Table 2), whereas the concentrations decreased on average by -11.8 (95\% Cl: $-7.1--16.6) \mu \mathrm{g} / \mathrm{mL}$ and $-3.6(95 \% \mathrm{Cl}:-1.1--6.2) \mu \mathrm{g} / \mathrm{mL}$ with each following dose in the tapering phase of the 3- and 4-week intervals, respectively (Figure 2C and 2D, Table 2). However, only three patients had a follow-up with five or more infusions at 3- and 4-week intervals. At 3-week intervals, the eculizumab levels remained relatively high in P4 and within/slightly above the target range of $50-100 \mu \mathrm{g} / \mathrm{mL}$ throughout the observed periods of 10 and 11 intervals (30 and 33 weeks) in P2 and P3, respectively. At 4-week intervals, the eculizumab levels remained within the recommended range throughout 12 intervals (48 weeks) of therapy for P4 only.

Body weight had a small effect on eculizumab level in the induction period only -3.9 (95\% Cl: $-1.8--6.0) \mu \mathrm{g} / \mathrm{mL}$ per $\mathrm{kg}$, while age did not have an effect in any of the intervals.

The number of available data points at 5-week intervals did not allow statistical analysis. However, four of the five patients analyzed still attained the eculizumab target concentration after the first 5-week interval and P10 also attained the target range after the second 5-week interval (Figure 2E). 


\section{Complement inhibition under eculizumab treatment}

All samples that were measured in our study and reached the eculizumab target level of $50 \mu \mathrm{g} / \mathrm{mL}$ had a $\mathrm{CH} 50$ value $\leq 12 \%$ (detection limit 10\%) (Figure 4).

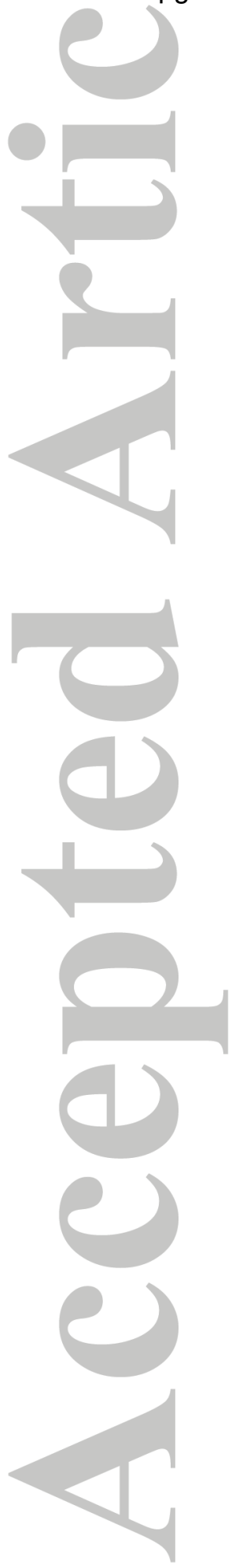




\section{Discussion}

In this report we, to our knowledge, for the first time performed a systematic analysis

of eculizumab levels in patients, receiving the drug with various (standard and extended) infusion intervals.

In the induction phase (1-week intervals) we have observed a weight-dependent increase in eculizumab concentration with each following infusion, which is consistent with the half-life of eculizumab in aHUS patients reported as 7.8-19.5 days [41], http://www.ema.europa.eu/ema/. In two patients with high initial sC5b-9 levels, the values were below the target range one week after the first infusion. Previous report investigating efficacy of eculizumab treatment in patients with TMA after hematopoietic stem cell transplantation indicated that patients with higher levels of sC5b-9 complex before treatment have faster initial eculizumab clearance [43]. Interestingly, recent study indicated that eculizumab is able to bind to $\mathrm{sC} 5 \mathrm{~b}-9$ in vitro, which suggests that part of the drug may be scavenged into a complex with sC5b-9 at first infusion [42]. Elevated sC5b-9 is a known biomarker in aHUS and thus should be taken into consideration in design of future personalized eculizumab protocols [44, 45].

In the maintenance phase (2-week intervals) the levels of eculizumab had reached extremely high values of up to $772 \mu \mathrm{g} / \mathrm{mL}$. Considerable excess of eculizumab in patients was also previously reported by our and other studies [32, 40-42]. Interestingly, one of the patients (P11) who had a severe pneumonia did not attain minimal target value of eculizumab and complete complement inhibition at the second infusion at 2-week interval. The reason of this is not clear, however, since levels of sC5b-9 have been shown to influence eculizumab pharmacokinetics, inflammation and increased rate of complement activation may have caused lower eculizumab concentration in this patient.

Although most patients attain eculizumab levels far above the recommended range at 2-week intervals, P11 illustrates that in some patients with ongoing active disease standard 
eculizumab dosing may be not sufficient for complement blockade and this should be taken into consideration when applying extended eculizumab regimen.

Interestingly, P11 developed severe pneumonia while having sub-therapeutic eculizumab levels. Although it was not a meningococcal infection, for which the terminal complement pathway is especially important, this case emphasizes the need to evaluate how much of residual complement activity is necessary to decrease risk of infection and how much of this activity may be safely allowed without risk of an aHUS relapse.

No significant correlation between eculizumab levels and number of received infusions was observed in the maintenance phase. Previously in another study, weight of the patient was correlated to the median trough concentration in a small group of aHUS patients $(n=7)$, which was not reproduced in our study [41]. Nevertheless, with the exception for P11, all other patients attained target eculizumab levels at all 2-week infusion intervals that were analyzed.

During tapering of the treatment with extended intervals of 3- and 4- weeks, statistically significant decrease in eculizumab concentration was observed with each infusion. This indicates, that complement blockade may remain adequate at the first infusions of the extended intervals, but diminish overtime.

However, at 3-week interval, in two patients (P2, P3) eculizumab recommended range of $50-100 \mu \mathrm{g} / \mathrm{mL}$ was attained and complement was efficiently blocked throughout the observed period of 10 and 11 intervals (30 and 33 weeks), respectively. At 4-week interval for one other patient (P4), the eculizumab levels remained within the recommended range and complement was completely blocked throughout 12 intervals (48 weeks) of therapy. Interestingly, of the three pediatric patients, who were able to sustain optimal eculizumab concentration in extended intervals, two (P3, P4) were treated with a standard adult dosage $(1200 \mathrm{mg})$ and one (P2) with standard pediatric dosage (300 mg) per infusion based on their body weight (Table 1). These three cases illustrate how monitoring of eculizumab concentrations and complement inhibition allows to establish optimal infusion intervals which can be maintained over a long period of time for patients on adult and pediatric regimen. 
Furthermore, our data indicate that at 5-week intervals attainment of target eculizumab range is possible at least for one or two infusions.

Overall, quite high inter-patient variability has been observed. In our manuscript we analyzed influence of body weight and age on the eculizumab levels in individual patients. Moreover, levels of the sC5b-9 complex may play a role in eculizumab clearance after the first eculizumab infusion and may contribute to variability. To facilitate individualized treatment options, inter-patient variability should be investigated further in the future.

Our findings suggest that C5 concentrations vary considerably among the patients, more than among the controls. Our previous data using C5 deficient serum indicated that even a $1-2 \%$ of unblocked C5 in serum may already lead to measurable complement activity [32]. Thus, when fine-tuning the eculizumab therapy based on patient's needs, C5 concentration may be important to take into account.

In our previous work, we measured eculizumab-C5 complexes in patients, which in the situation of substantial eculizumab excess, as was observed in that study, provided a good indication of C5 levels. The data indicated some fluctuation of eculizumab-C5 complexes [32] during the course of therapy. Relevance of these findings for personalized treatment should be investigated further.

In this study only dosing intervals have been varied. However, in the future also options for the adjustment of the administered eculizumab dosage should be investigated. To this end, proportionality across the recommended dosing range of $50-100 \mu \mathrm{g} / \mathrm{mL}$ should be addressed at various diseases stages. Current knowledge on pharmacokinetics and pharmacodynamics indicates that eculizumab is bound to $\mathrm{C} 5$ and $\mathrm{sC} 5 \mathrm{~b}-9$ in vitro [42]. In vivo deposition on other ligands, including surface of blood cells, endothelium, etc. is also possible. It may interfere with drug's complement inhibiting activity and thus should be taken into account when lowering the treatment dose.

In this study three samples with $93-250 \mu \mathrm{g} / \mathrm{mL}$ of eculizumab and residual $11-12 \%$ activity in $\mathrm{CH} 50$ assay were observed (Figure 4). These findings are in line with previous work, where the authors also observed several samples with eculizumab $>99 \mu \mathrm{g} / \mathrm{mL}$ (which 
they use as a target reference) and $\mathrm{CH} 50>10 \%$ [43]. Interestingly, recent data point out that eculizumab inhibition of complement may not always be complete and even high drug levels would not always completely block complement in an in vitro assay [46]. Clinical importance of such residual complement activity in aHUS should be further investigated.

Thus, in this study we performed a thorough analysis of data of patients who were treated with eculizumab and where standard and extended intervals between infusions were applied. Our data indicate that eculizumab accumulates in the body during 1-week intervals (depending on the body weight and possibly initial sC5b-9 concentration) remains stable at 2-week intervals, and diminishes during 3- and 4- week intervals. Importantly, extended 3and 4- week intervals may be optimal for complete complement inhibition in a considerable subset of pediatric and adult patients, as shown in this study. Our data indicate that even at 5-week intervals at least some of the patients were able to sustain optimal eculizumab concentration and complement inhibition, at least for a short time. As our study included a relatively limited number of patients, further optimization of treatment intervals in aHUS should be evaluated in a larger patient cohort.

The results of this study have laid the base to formulate the Dutch guideline for the restrictive treatment regimen of aHUS patients that is currently being introduced in the Netherlands. In the future, it will help to design correct individualized dosing schemes for patients in acute aHUS that require continuous complement blockade, as well as for patients in remission for whom therapy is being discontinued by using gradual extension of intervals. That will help to save costs and make this efficient medication more accessible to the patients.

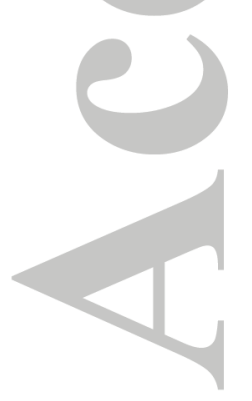




\section{Methods}

\section{Patient cohort}

The 11 patients, described in this study were diagnosed with aHUS and received treatment at the Radboud university medical center, Nijmegen, The Netherlands. Eculizumab was administered in the dosages that are recommended by the current EMA guidelines (http://www.ema.europa.eu/ema/). The current treatment schedule is split into two phases: the initial phase (weekly infusions for up to 4 weeks for patients $\geq 40 \mathrm{~kg}$ body weight), followed by the maintenance phase (life-long infusions every two weeks). For pediatric patients ( $<18$ years old, $<40 \mathrm{~kg}$ ), the dosage regimen is adjusted to body weight. The other patients (pediatric and adult) receive the drug according to the schedule for adults.

In this report, in patients who have reached remission (normalized hematological parameters and improved/stable renal function), the therapy was reevaluated and intervals between infusions were gradually extended (while keeping maintenance infusion doses unchanged) or therapy was discontinued (Table 1). In two patients, eculizumab treatment was discontinued without extension of dosage intervals beyond two weeks due to an allergic reaction (patient $8(\mathrm{P} 8)$ ) and severe pneumonia $(\mathrm{P} 11)$. In nine patients $\geq 40 \mathrm{~kg}$, the eculizumab dose was $1200 \mathrm{mg}$ in the maintenance/tapering phase and for the two children with body weight of $15 \mathrm{~kg}(\mathrm{P} 2)$ and $11 \mathrm{~kg}(\mathrm{P} 9)$, the dose was $300 \mathrm{mg}$.

During tapering/discontinuation of therapy, all patients and/or their caregivers were strictly instructed to contact their physician in case of any signs of infections, generalized malaise, fever, hematuria, edema, oliguria, paleness or other signs indicating recurrence of aHUS. In case of signs of aHUS recurrence (which did not take place during the described period), eculizumab would be restarted immediately.

\section{Collection of blood samples}

Blood samples from healthy adult controls and patients just before each eculizumab infusion were collected and placed immediately on ice (ethylenediamineteraacetic acid (EDTA) blood) or allowed to clot for 30-40 minutes ( $\mathrm{min}$ ) at room temperature (whole blood). 
EDTA plasma and serum samples were collected by centrifugation (10 min, $2000 \times \mathrm{g}, 4^{\circ} \mathrm{C}$ ), aliquoted and stored at $-80^{\circ} \mathrm{C}$.

\section{Assay for detection of serum eculizumab concentration}

Eculizumab detection was performed using in-house enzyme-linked immunosorbent assay (ELISA). To capture eculizumab, 96-well plates (Greiner Microlon® 600 High Binding, Sigma-Aldrich, Zwijndrecht, The Netherlands) were coated with $0.5 \mu \mathrm{g} /$ well of purified C5 (Calbiochem ${ }^{\circledR}$, San Diego, CA, USA) diluted in carbonate buffer $(\mathrm{pH}=9.6)$ overnight at $4^{\circ} \mathrm{C}$. The wells were washed with phosphate buffered saline (PBS) supplemented with $0.05 \%$ Tween-20 (PBST) (Sigma-Aldrich) after this and following steps. The plates were blocked with SuperBlock (PBS) Blocking Buffer (Thermo Fisher Scientific, Waltham, MA, USA) 30 min at room temperature (RT) and then $45 \mathrm{~min}$ at RT with PBS, supplemented with $1 \%$ bovine serum albumin (PBS/BSA). Serum samples were diluted in PBS/BSA and dose response standards were prepared by adding eculizumab (Alexion Pharmaceuticals, Cheshire, CT, USA) to PBS/BSA. Samples and standards were added to the wells and incubated for 2 hours (h) at RT. Eculizumab detection was performed using incubation with Peroxidase AffiniPure Goat Anti-Human IgG (H+L) (Jackson ImmunoResearch Laboratories Inc., West Grove, PA, USA) diluted in PBST for $2 \mathrm{~h}$ at RT, o-phenylenediamine dihydrochloride tablets (Dako, Hevelee, Belgium) were added as a substrate. The reaction was stopped with $2 \mathrm{M} \mathrm{H}_{2} \mathrm{SO}_{4}$, and samples were measured in a 96-well microtiter plate reader at $490 \mathrm{~nm}$.

Dilution linearity of the test was analyzed by measuring serum containing $383 \mu \mathrm{g} / \mathrm{mL}$ eculizumab and had coefficient of variation (CV) of $10.5 \%$ over the range of a standard curve. The detection limit of an assay was $8 \mu \mathrm{g} / \mathrm{mL}$ for undiluted samples. The assay showed variation coefficients of $2.9 \%$ (intra-assay, $352 \mu \mathrm{g} / \mathrm{mL}$ ) and $5.2 \%$ (inter-assay, $328 \mu \mathrm{g} / \mathrm{mL}$ ). Recovery was determined in 20 control samples spiked with $50 \mu \mathrm{g} / \mathrm{mL}(68 \pm 31 \%), 75 \mu \mathrm{g} / \mathrm{mL}$ $(66 \pm 25 \%)$ and $150 \mu \mathrm{g} / \mathrm{mL}(74 \pm 17 \%)$. 


\section{C5 assay}

C5 concentrations were measured in available sera or EDTA plasma, that were collected from 10 aHUS patients in acute phase, before the start of eculizumab therapy and from nine healthy volunteers. The C5 levels were measured using Human Complement C5 ELISA Kit (Abcam, Cambridge, UK), according to manufacturer's instructions.

\section{Complement assays}

The concentrations of fluid phase terminal C5b-9 complement complex (sC5b-9) in seven available EDTA plasma samples were quantified using ELISA, as previously described in detail by Bergseth etal. [47]. International complement standard 2 was used for quantification of sC5b-9 in complement activation units per $\mathrm{mL}(\mathrm{CAU} / \mathrm{mL})$.

Complement activity was accessed in serum samples by determining the activity of classical complement route $(\mathrm{CH} 50)$ using an ELISA method adapted from [48]. Briefly, polyclonal goat (affinity purified) anti-human IgM (Cappel, Bioconnect Life Sciences, Huissen, The Netherlands) was coated in 96-wells plates (Nunc Maxisorb plates, Nunc, Roskilde, Denmark) at a concentration of $0.2 \mu \mathrm{g} /$ well in carbonate buffer ( $\mathrm{pH} 9.6)$, overnight at $4^{\circ} \mathrm{C}$. The plates were washed with Tris buffered saline (TBS) containing $0.05 \%$ Tween-20 (TBST) after each step. Residual binding sites were blocked by incubation with $10 \mathrm{mM}$ TBS containing $1 \%$ gelatin (BD Difco ${ }^{\mathrm{TM}}$, Franklin Lakes, New Jersey, USA) for $1 \mathrm{~h}$ at RT. After blocking, $0.25 \mu \mathrm{g} /$ well IgM (Fitzgerald Industries International, Acton, MA, USA) diluted in TBST was added and incubated for $1 \mathrm{~h}$ at RT. Next, standards (human pooled serum) and patient serum samples diluted in cold buffer containing $10 \mathrm{mM}$ Tris, $84 \mathrm{mM} \mathrm{NaCl}, 0.5 \mathrm{mM}$ $\mathrm{MgCl}_{2}, 2 \mathrm{mM} \mathrm{CaCl}$ and $0.1 \%$ gelatin $(\mathrm{pH} 7.5)$, were added per well and incubated for $1 \mathrm{~h}$ at $37^{\circ} \mathrm{C}$. Complement binding was detected using a monoclonal mouse-anti-human C $5 \mathrm{~b}-9$ antibody (Dako) diluted in TBST and incubated for $1 \mathrm{~h}$ at RT. After washing, alkaline phosphatase labeled goat anti-mouse IgG (Dako) in TBST was added for $1 \mathrm{~h}$ at RT. Alkaline phosphatase activity was revealed following incubation with 4-nitrophenyl phosphate 
disodium salt hexahydrate as a substrate (Sigma-Aldrich). The reaction was stopped with 2 $\mathrm{M} \mathrm{NaOH}$, and samples were measured in a 96-well microtiter plate reader at $450 \mathrm{~nm}$.

The reference value of $\mathrm{CH} 50$ of $67-149 \%$ was calculated with the results of 50 healthy donors.

\section{Statistical analyses}

GraphPad Prism 5 for Windows (GraphPad Software Inc., La Jolla, CA, USA) was used to plot eculizumab levels against treatment intervals, and sC5b-9 complex concentrations and $\mathrm{CH} 50 \%$ against eculizumab levels.

If a patient did not reach the minimal target level of $50 \mu \mathrm{g} / \mathrm{mL}$ eculizumab $\geq 1$ time in a treatment interval, he/she was considered not to have attained the target level for that interval. Following, the percentages of patients not reaching the eculizumab target level were plotted against treatment interval.

To evaluate changes in eculizumab concentrations by the number of infusions within a treatment interval, we used IBM Statistics 22.0 for Windows (IBM SPSS Inc., Chicago, IL, USA). For each treatment interval, we plotted eculizumab levels against infusion number and estimated the increase or decrease in eculizumab level per infusion with $95 \%$ confidence intervals $(\mathrm{Cl})$, using linear mixed models with infusion numbers as fixed and patients as random effects. Body weight (in $\mathrm{kg}$ ) and age (in years) were included in these models as potential confounders. 


\section{Study highlights}

\section{What is the current knowledge on the topic?}

Under the standard treatment regimen eculizumab levels in patients are very high. This increases the risk of side effects, such as meningococcal infection, and is associated with extremely high costs of the treatment. Pharmacokinetic and pharmacodynamic data to develop individualized eculizumab treatment schemes are limited.

What question did this study address?

Here we analyzed eculizumab serum concentrations in aHUS patients treated with standard and extended eculizumab infusion intervals.

\section{What this study adds to our knowledge?}

This is the first report analyzing eculizumab concentration in patients undergoing individualized treatment.

\section{How this might change clinical pharmacology or translational science?}

Our data provide essential insight for better optimization of eculizumab dosing schemes and lessening of therapy burden for the patients and costs of the treatment. 


\section{Acknowledgments}

We thank Nicolai Grebenchtchikov and Anneke Geurts-Moespot for their advice during development of the eculizumab ELISA and Corrie de Kat Angelino for technical support. This work was supported by grants from the Dutch Kidney Foundation (130l116, KFB 11.007, IP 10.22, 160KKO1), European Renal Association - European Dialysis and Transplantation Association (ERA STF 138-2013, ERA LTF 203-2014) and European Society for Pediatric Nephrology (2014.03).

\section{Disclosures}

Dr. N.C.A.J. van de Kar and Prof. Dr. J.F.M. Wetzels are both members of the international advisory board of Alexion. Prof. Dr. J.F.M. Wetzels received a grant from Alexion.

\section{Author contributions}

E.V. wrote the manuscript; E.V., F.S., J.W., N.v.d.K., and L.v.d.H. designed the research; E.V., K.W., R.v.d.M., T.v.d.V., J.G., J.W., N.v.d.K., and L.v.d.H. performed the research; E.V., K.W., R.v.d.M., N.R., F.S., R.B., J.W., N.v.d.K., and L.v.d.H. analyzed the data; E.V. contributed new reagents/analytical tools.

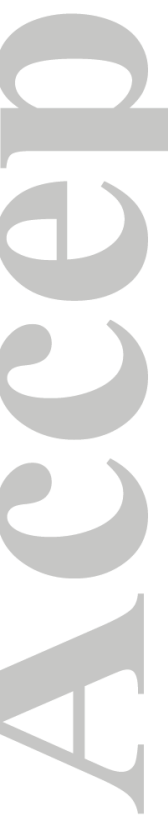




\section{References}

1. Noris M, Remuzzi G. Atypical hemolytic-uremic syndrome. N Engl J Med 2009;361(17):16761687

2. Loirat C, Fremeaux-Bacchi V. Atypical hemolytic uremic syndrome. Orphanet J Rare Dis 2011;6:60

3. Westra D, Wetzels JF, Volokhina EB, et al. A new era in the diagnosis and treatment of atypical haemolytic uraemic syndrome. Neth J Med 2012;70(3):121-129

4. Fremeaux-Bacchi V, Fakhouri F, Garnier A, et al. Genetics and outcome of atypical hemolytic uremic syndrome: a nationwide French series comparing children and adults. Clin J Am Soc Nephrol 2013;8(4):554-562

5. Verhave JC, Wetzels JF, van de Kar NC. Novel aspects of atypical haemolytic uraemic syndrome and the role of eculizumab. Nephrol Dial Transplant 2014;29 Suppl 4:iv131-141

6. Nester CM, Barbour T, de Cordoba SR, et al. Atypical aHUS: State of the art. Mol Immunol 2015;67(1):31-42

7. Stahl AL, Vaziri-Sani F, Heinen S, et al. Factor $\mathrm{H}$ dysfunction in patients with atypical hemolytic uremic syndrome contributes to complement deposition on platelets and their activation. Blood 2008;111(11):5307-5315

$8 . \quad$ Lehtinen MJ, Rops AL, Isenman DE, et al. Mutations of factor $\mathrm{H}$ impair regulation of surfacebound $\mathrm{C} 3 \mathrm{~b}$ by three mechanisms in atypical hemolytic uremic syndrome. J Biol Chem 2009;284(23):15650-15658

9. $\quad$ Fremeaux-Bacchi V, Dragon-Durey MA, Blouin J, et al. Complement factor I: a susceptibility gene for atypical haemolytic uraemic syndrome. J Med Genet 2004;41(6):e84

10. Richards A, Kemp EJ, Liszewski MK, et al. Mutations in human complement regulator, membrane cofactor protein (CD46), predispose to development of familial hemolytic uremic syndrome. Proc Natl Acad Sci U S A 2003;100(22):12966-12971

11. Noris M, Brioschi S, Caprioli J, et al. Familial haemolytic uraemic syndrome and an MCP mutation. Lancet 2003;362(9395):1542-1547

12. Esparza-Gordillo J, Goicoechea de Jorge E, Buil A, et al. Predisposition to atypical hemolytic uremic syndrome involves the concurrence of different susceptibility alleles in the regulators of complement activation gene cluster in 1q32. Hum Mol Genet 2005;14(5):703-712

13. Caprioli J, Noris M, Brioschi S, et al. Genetics of HUS: the impact of MCP, CFH, and IF mutations on clinical presentation, response to treatment, and outcome. Blood 2006;108(4):12671279

14. Fremeaux-Bacchi V, Miller EC, Liszewski MK, et al. Mutations in complement C3 predispose to development of atypical hemolytic uremic syndrome. Blood 2008;112(13):4948-4952

15. Roumenina LT, Frimat M, Miller EC, et al. A prevalent C3 mutation in aHUS patients causes a direct C3 convertase gain of function. Blood 2012;119(18):4182-4191

16. Volokhina $E$, Westra $D$, Xue X, et al. Novel C3 mutation p.Lys65GIn in aHUS affects complement factor $\mathrm{H}$ binding. Pediatr Nephrol 2012;27(9):1519-1524

17. Goicoechea de Jorge E, Harris CL, Esparza-Gordillo J, et al. Gain-of-function mutations in complement factor B are associated with atypical hemolytic uremic syndrome. Proc Natl Acad Sci U S A 2007;104(1):240-245

18. Delvaeye M, Noris M, De Vriese A, et al. Thrombomodulin mutations in atypical hemolyticuremic syndrome. N Engl J Med 2009;361(4):345-357

19. Jozsi M, Licht $\mathrm{C}$, Strobel $\mathrm{S}$, et al. Factor $\mathrm{H}$ autoantibodies in atypical hemolytic uremic syndrome correlate with CFHR1/CFHR3 deficiency. Blood 2008;111(3):1512-1514

20. Venables JP, Strain L, Routledge D, et al. Atypical haemolytic uraemic syndrome associated with a hybrid complement gene. PLoS Med 2006;3(10):e431

21. Challis RC, Araujo GS, Wong EK, et al. A De Novo Deletion in the Regulators of Complement Activation Cluster Producing a Hybrid Complement Factor H/Complement Factor H-Related 3 Gene in Atypical Hemolytic Uremic Syndrome. J Am Soc Nephrol 2016;27(6):1617-1624 
22. Skerka C, Chen Q, Fremeaux-Bacchi V, et al. Complement factor $\mathrm{H}$ related proteins (CFHRs). Mol Immunol 2013;56(3):170-180

23. Rother RP, Rollins SA, Mojcik CF, et al. Discovery and development of the complement inhibitor eculizumab for the treatment of paroxysmal nocturnal hemoglobinuria. Nat Biotechnol 2007;25(11):1256-1264

24. Waters AM, Licht $C$. aHUS caused by complement dysregulation: new therapies on the horizon. Pediatr Nephrol 2011;26(1):41-57

25. Zuber J, Fakhouri F, Roumenina LT, et al. Use of eculizumab for atypical haemolytic uraemic syndrome and C3 glomerulopathies. Nat Rev Nephrol 2012;8(11):643-657

26. Wong EK, Goodship TH, Kavanagh D. Complement therapy in atypical haemolytic uraemic syndrome (aHUS). Mol Immunol 2013;56(3):199-212

27. Legendre CM, Licht C, Muus $\mathrm{P}$, et al. Terminal complement inhibitor eculizumab in atypical hemolytic-uremic syndrome. N Engl J Med 2013;368(23):2169-2181

28. Bouts A, Monnens L, Davin JC, et al. Insufficient protection by Neisseria meningitidis vaccination alone during eculizumab therapy. Pediatr Nephrol 2011;26(10):1919-1920

29. Struijk GH, Bouts AH, Rijkers GT, et al. Meningococcal sepsis complicating eculizumab treatment despite prior vaccination. Am J Transplant 2013;13(3):819-820

30. Ariceta G, Besbas N, Johnson S, et al. Guideline for the investigation and initial therapy of diarrhea-negative hemolytic uremic syndrome. Pediatr Nephrol 2009;24(4):687-696

31. Taylor CM, Machin S, Wigmore SJ, et al. Clinical practice guidelines for the management of atypical haemolytic uraemic syndrome in the United Kingdom. Br J Haematol 2010;148(1):37-47

32. Volokhina EB, van de Kar NC, Bergseth G, et al. Sensitive, reliable and easy-performed laboratory monitoring of eculizumab therapy in atypical hemolytic uremic syndrome. Clin Immunol 2015;160(2):237-243

33. Carr R, Cataland SR. Relapse of aHUS after discontinuation of therapy with eculizumab in a patient with aHUS and factor H mutation. Ann Hematol 2013;92(6):845-846

34. Ardissino G, Testa S, Possenti I, et al. Discontinuation of Eculizumab Maintenance Treatment

for Atypical Hemolytic Uremic Syndrome: A Report of 10 Cases. Am J Kidney Dis 2014;64(4):633-637

35. Pu JJ, Sido A. Successful discontinuation of eculizumab therapy in a patient with aHUS. Ann Hematol 2014;93(8):1423-1425

36. Wetzels JF, van de Kar NC. Discontinuation of eculizumab maintenance treatment for atypical hemolytic uremic syndrome. Am J Kidney Dis 2015;65(2):342

37. Habbig S, Bergmann C, Weber LT. Discontinuation of Eculizumab in a Patient With Atypical Hemolytic Uremic Syndrome Due to a Mutation in CFH. Am J Kidney Dis 2016;67(3):532-533

38. Toyoda H, Wada H, Miyata T, et al. Disease Recurrence After Early Discontinuation of Eculizumab in a Patient With Atypical Hemolytic Uremic Syndrome With Complement C3 I1157T Mutation. J Pediatr Hematol Oncol 2016;38(3):e137-139

39. Fakhouri F, Fila M, Provot F, et al. Pathogenic Variants in Complement Genes and Risk of Atypical Hemolytic Uremic Syndrome Relapse after Eculizumab Discontinuation. Clin J Am Soc Nephrol 2016;12(1):50-59

40. Greenbaum LA, Fila M, Ardissino G, et al. Eculizumab is a safe and effective treatment in pediatric patients with atypical hemolytic uremic syndrome. Kidney Int 2016;89(3):701-711

41. Gatault $P$, Brachet $G$, Ternant $D$, et al. Therapeutic drug monitoring of eculizumab: Rationale for an individualized dosing schedule. MAbs 2015;7(6):1205-1211

42. Wehling $\mathrm{C}$, Amon $\mathrm{O}$, Bommer $\mathrm{M}$, et al. Monitoring of complement activation biomarkers and eculizumab in complement-mediated renal disorders. Clin Exp Immunol 2016;187(2):304-315

43. Jodele S, Fukuda T, Mizuno K, et al. Variable Eculizumab Clearance Requires Pharmacodynamic Monitoring to Optimize Therapy for Thrombotic Microangiopathy after Hematopoietic Stem Cell Transplantation. Biol Blood Marrow Transplant 2016;22(2):307-315

44. Volokhina EB, Westra D, van der Velden TJ, et al. Complement activation patterns in atypical haemolytic uraemic syndrome during acute phase and in remission. Clin Exp Immunol 2015;181(2):306-313 
45. Bu F, Meyer NC, Zhang Y, et al. Soluble c5b-9 as a biomarker for complement activation in atypical hemolytic uremic syndrome. Am J Kidney Dis 2015;65(6):968-969

46. Harder MJ, Kuhn N, Schrezenmeier $\mathrm{H}$, et al. Incomplete inhibition by eculizumab: mechanistic evidence for residual C5 activity during strong complement activation. Blood 2017;129(8):970-980

47. Bergseth $G$, Ludviksen JK, Kirschfink M, et al. An international serum standard for application in assays to detect human complement activation products. Mol Immunol 2013;56(3):232-239

48. Roos A, Bouwman LH, Munoz J, et al. Functional characterization of the lectin pathway of complement in human serum. Mol Immunol 2003;39(11):655-668
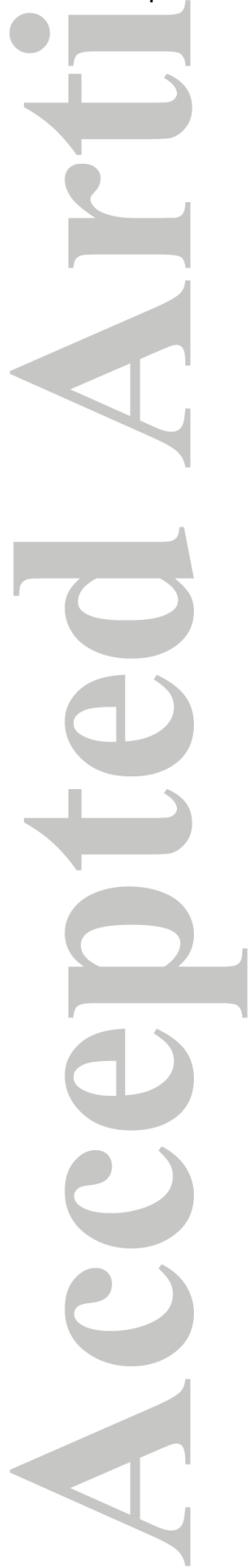


\section{Figure legends}

Figure 1. Serum eculizumab concentrations in aHUS cohort. A. Eculizumab trough levels in 11 patients in the induction (weekly), maintenance (2-weekly) and tapering (every 3-8 weeks) phases of therapy (Table 1). From one patient (P1), samples were also collected after discontinuation of treatment (at 14, 20, 26 weeks). The dashed lines indicate the recommended range of eculizumab levels of $50-100 \mu \mathrm{g} / \mathrm{mL}$. B. Percentages of patients that attained the trough eculizumab level of $50 \mu \mathrm{g} / \mathrm{mL}$ in all measured samples in an interval are indicated in white, whereas percentages of patients with a value $<50 \mu \mathrm{g} / \mathrm{mL}$ in at least one sample are indicated in gray. Total numbers of patients analyzed for each interval are shown above the columns.

Figure 2. Eculizumab concentrations in individual patients during infusions with standard and extended intervals. Eculizumab trough levels in 11 patients before next eculizumab infusion in the induction (1- week intervals, A), maintenance (2-week intervals, B) and tapering (3-, 4- and 5-week intervals, C, D and E) phases of therapy. The dashed lines indicate the recommended range of eculizumab levels of $50-100 \mu \mathrm{g} / \mathrm{mL}$. Observed effects $\left({ }^{*}\right)$ of each following infusion or weight of a patient $(\mathrm{kg})$ on eculizumab concentration as presented in Table 2 are indicated.

\section{Figure 3. SC5b-9 complex concentration at baseline and trough eculizumab levels after} the first infusion. Seven patients $(\mathrm{P} 1, \mathrm{P} 3, \mathrm{P} 4, \mathrm{P} 7, \mathrm{P} 9, \mathrm{P} 10, \mathrm{P} 11)$ of whom EDTA plasma was available were included. The dashed lines indicate the recommended range of eculizumab levels of $50-100 \mu \mathrm{g} / \mathrm{mL}$. Patients that did not attain the recommended eculizumab level after first infusion are indicated (P10 and P11).

\section{Figure 4. Complement inhibition during eculizumab treatment in aHUS patients.}

Complement activity ( $\mathrm{CH} 50 \%)$ data of the samples included in the study are presented as percentage of activity of normal human pooled serum. The dashed lines indicate the recommended range of eculizumab levels of $50-100 \mu \mathrm{g} / \mathrm{mL}$. 
A

Eculizumab levels
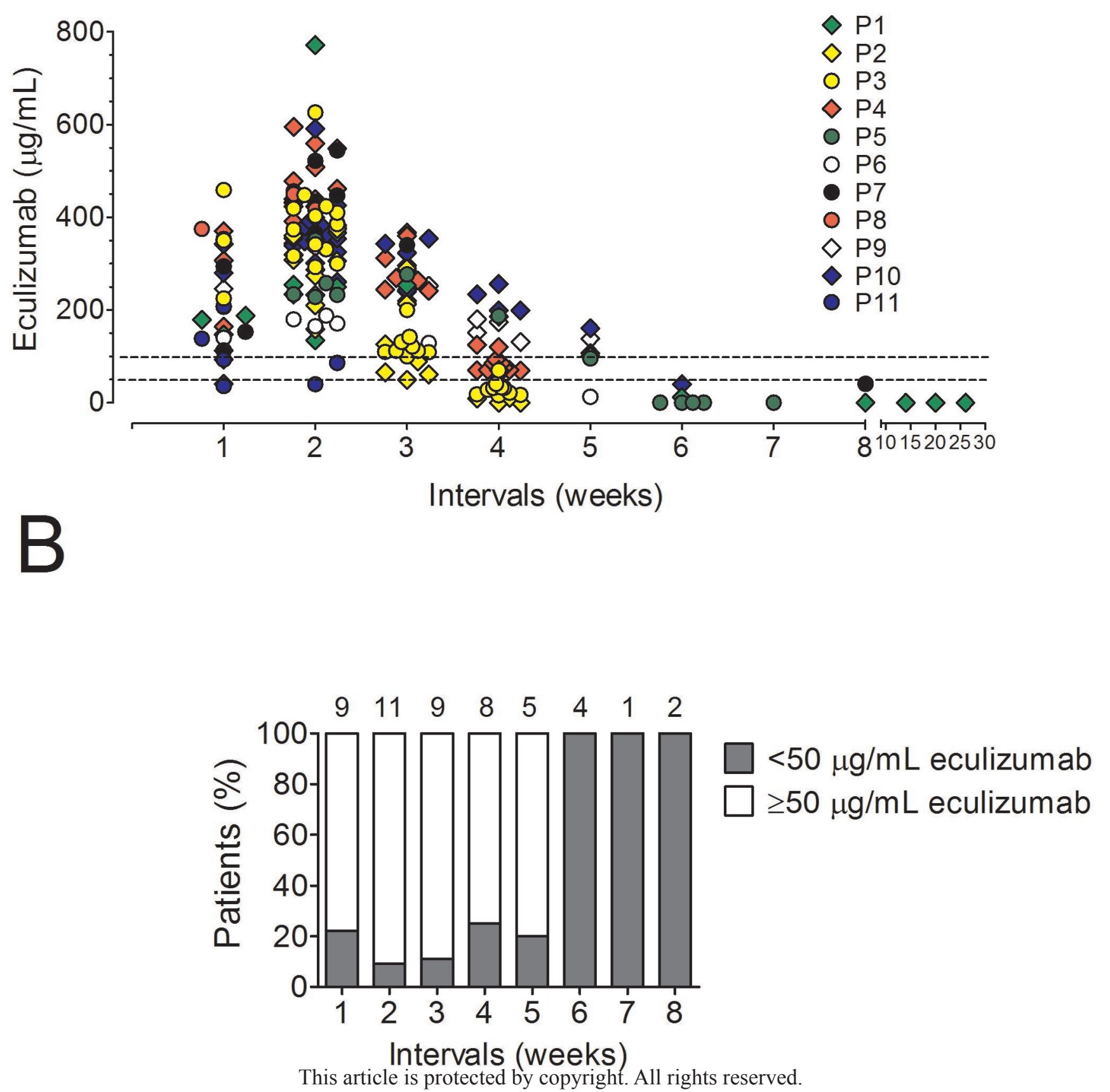
A

1-week interval

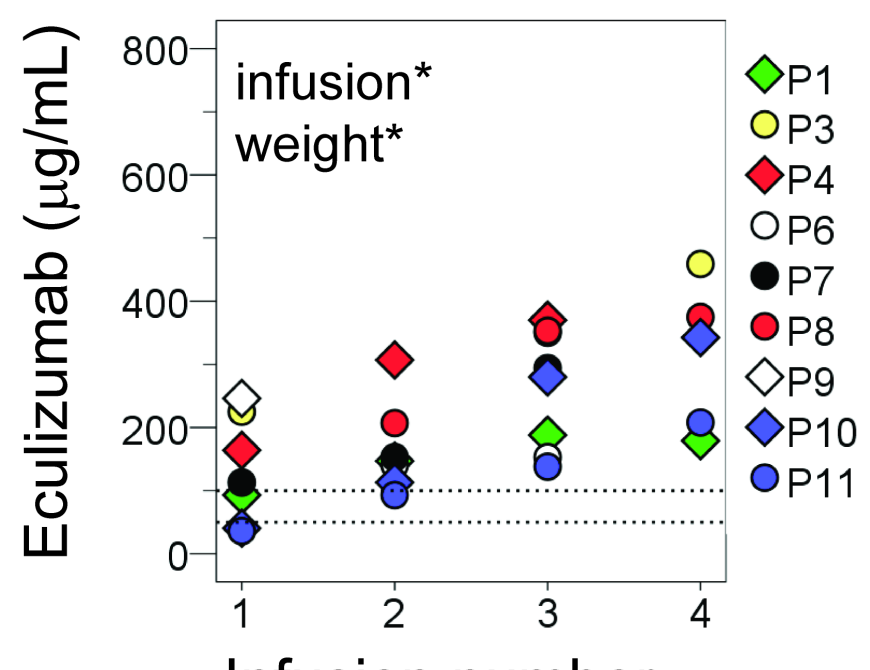

Infusion number

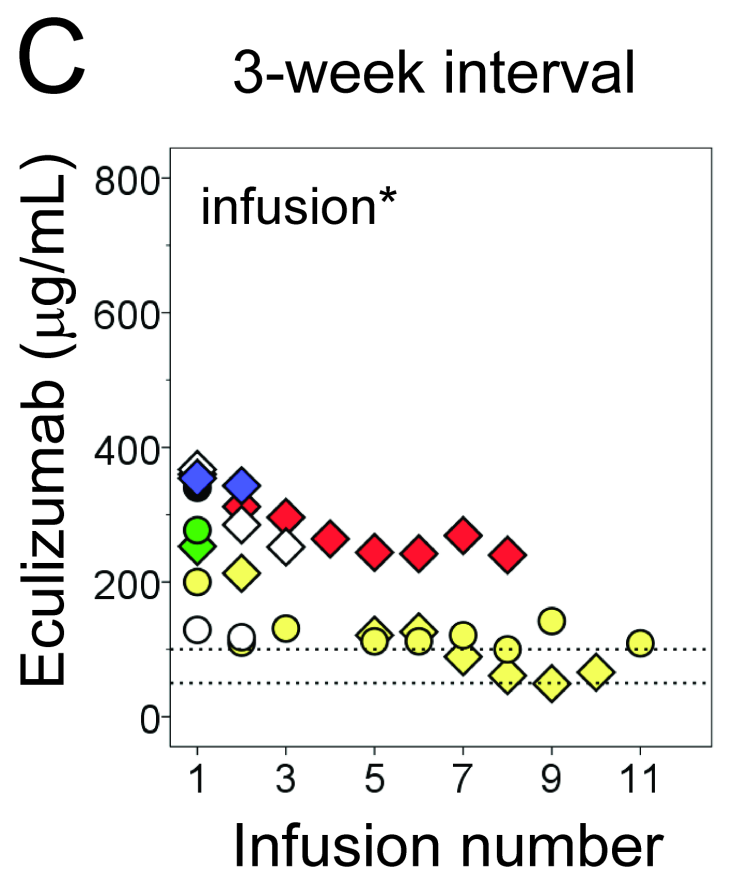

\section{5-week interval}

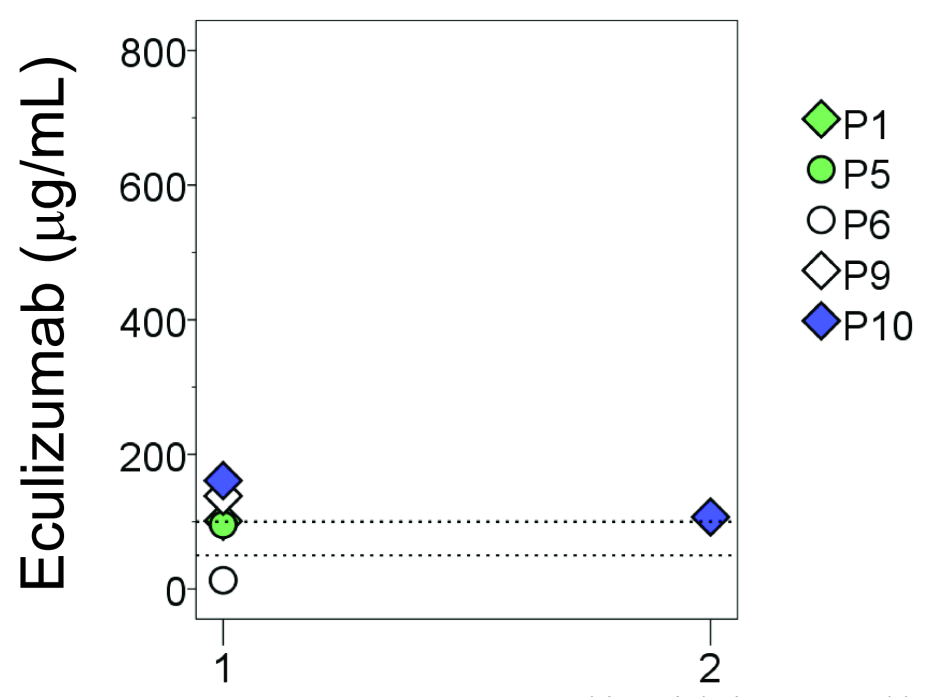

Infusion numb'erticle is protected by copyright. All rights reserved. 


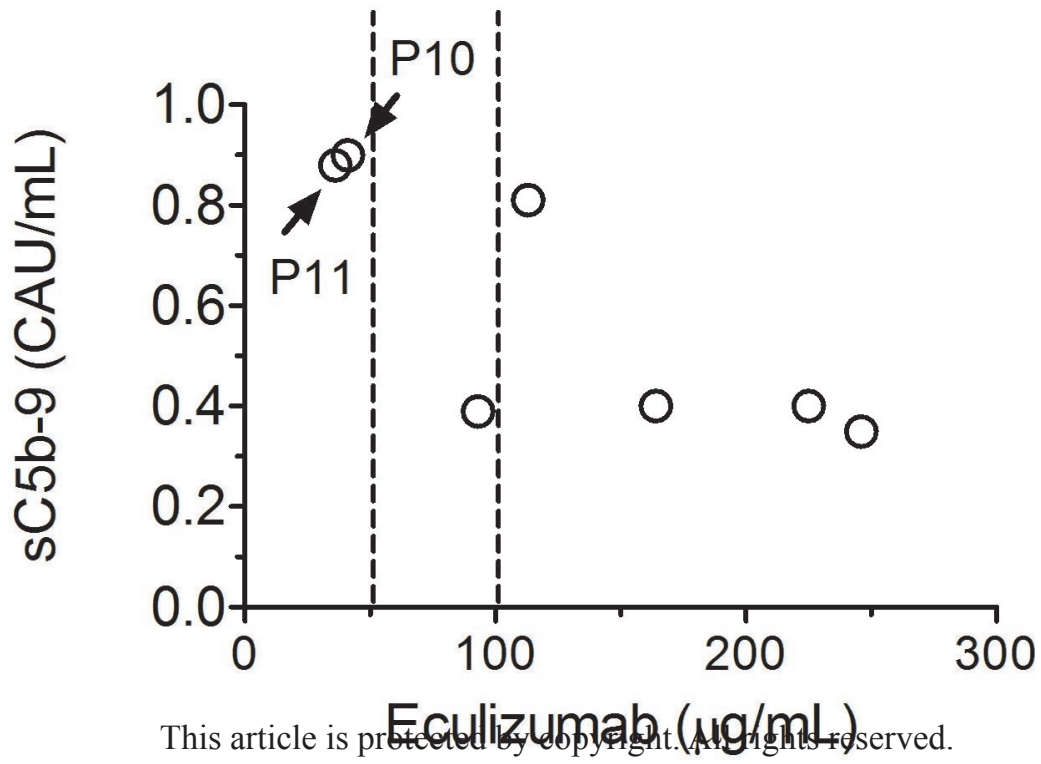




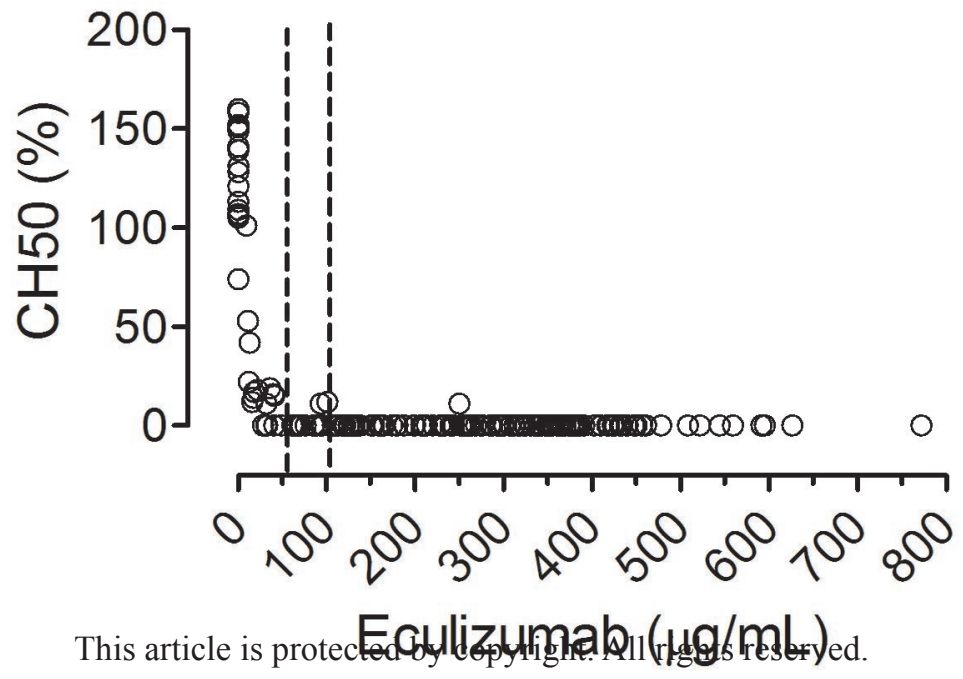


Table 1. Characteristics of aHUS patients.

\begin{tabular}{|c|c|c|c|c|c|c|}
\hline Patient & aHUS pathogenic change & $\begin{array}{l}\text { Gender } \\
\text { (F/M) }\end{array}$ & $\begin{array}{l}\text { Age at start } \\
\text { of the } \\
\text { observation } \\
\text { (years) }\end{array}$ & $\begin{array}{l}\text { Body weight } \\
\text { (kg) }\end{array}$ & $\begin{array}{l}\text { Studied dose } \\
\text { intervals } \\
\text { (weeks) }^{1}\end{array}$ & $\begin{array}{l}\text { Total } \\
\text { observation } \\
\text { period (months) }\end{array}$ \\
\hline P1 & CFH: c.1778T>A, p.Leu593Stop & $\mathrm{F}$ & 28 & 61 & $1-6,8,14,20,26$ & 15.5 \\
\hline P2 & $\begin{array}{l}\text { CFH: c.3572C>T (p.Ser1191Leu); } \\
\text { CFH: c.3590T>C (p.Val1197Ala) }\end{array}$ & M & 2 & 15 & $2-4$ & 24 \\
\hline P3 & Anti-CFH & M & 9 & 50 & $1-4$ & 28.5 \\
\hline P4 & $\mathrm{CFH} / \mathrm{CFH} 1$ hybrid protein & M & 11 & 45 & $1-4$ & 30.5 \\
\hline P5 & CFH: c.2572T>A (p.Trp858Arg) & $\mathrm{F}$ & 22 & 114 & $2-7$ & 12 \\
\hline P6 & CFH: c.2120delC & $\mathrm{F}$ & 45 & 71 & $1-6$ & 7 \\
\hline P7 & C3: c.481C>T (p.Arg161Trp) & $\mathrm{F}$ & 62 & 56 & $1-3,8$ & 8 \\
\hline $\mathbf{P 8}^{2}$ & C3: c.481C>T (p.Arg161Trp) & $\mathrm{F}$ & 11 & 46 & 1,2 & 2.5 \\
\hline P9 & No changes found & $M$ & 1.3 & 11 & $1-5$ & 11.5 \\
\hline P10 & CFH: c.1520-1G>A (splice site) & $\mathrm{F}$ & 44 & 53 & $1-7$ & 23.5 \\
\hline $\mathbf{P} 11^{2}$ & CFI: c.685T>C (p.Cys229Arg) & $\mathrm{F}$ & 21 & 85 & 1,2 & 2 \\
\hline
\end{tabular}

${ }^{1}$ All patients with body weight $\geq 40$ kilo were treated with four weekly infusions of $900 \mathrm{mg}$ at start of therapy, after that, starting from week 5 the patients were treated with $1200 \mathrm{mg}$. For P2 and P9 pediatric dosage was applied (600 mg infusion at first week, $300 \mathrm{mg}$ at week 2 and further). Samples were taken after the indicated intervals since last eculizumab infusion.

${ }^{2}$ Eculizumab treatment in these patients was discontinued during the study due to allergic reaction (P8) and severe pneumonia (P11). 
Table 2 Estimated effects of number of infusions on eculizumab concentration in serum per treatment interval.

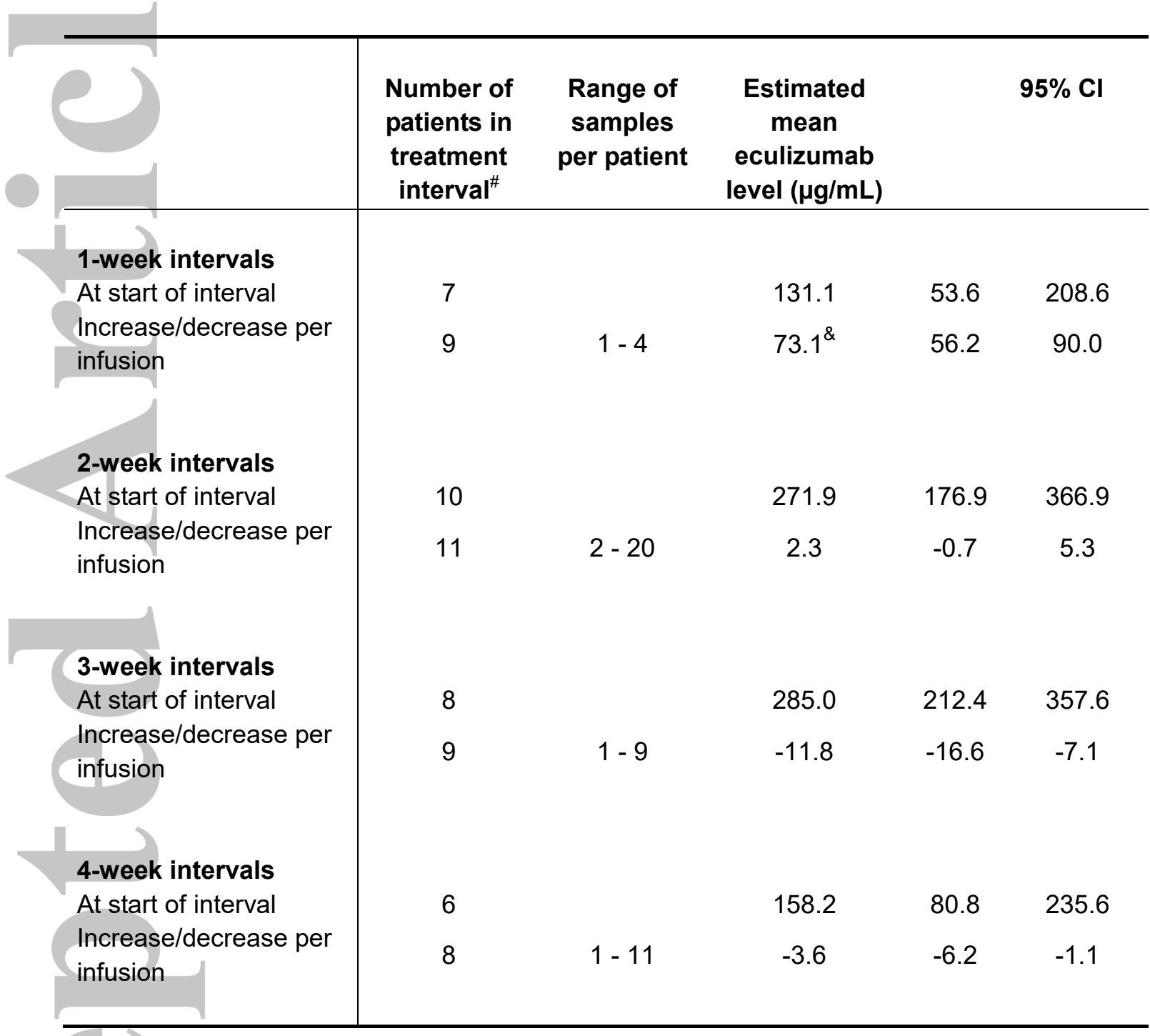

\# At the start of each interval, data were not collected from all patients

\& Adjusted for patient's body weight $(\mathrm{kg})$; weight and age did not influence the other estimates. 